\title{
Apport De La Stratigraphie Sequentielle Dans L'evolution Jurassique Du Moyen Atlas Central (Maroc)
}

Benjelloun Faiza, (Associate Professor)

Laboratoire Géoressources et Environnement /

Université sidi Mohamed Ben Abdellah, Maroc, Fez, Morocco

Fedan Bouazza, (Professor)

Scientific Institute, Rabat, Morocco

Anjjar Ahmed, (PhD Student)

Lahrach Abderrahim, (Professor)

Boukhir Mohammed, (Professor)

Chaouni Abdel-Ali, (Professor)

Laboratoire Géoressources et Environnement /

Université sidi Mohamed Ben Abdellah, Maroc, Fez, Morocco

doi: 10.19044/esj.2017.v13n21p155 URL:http://dx.doi.org/10.19044/esj.2017.v13n21p155

\begin{abstract}
The Jurassic evolution of the central Middle Atlas is governed by various factors (climate and eustatism). The use of the concepts of sequential stratigraphy in the regions of Tagnamas and Boulemane allowed to interpret the geometry and the vertical sequence of sedimentary sets. Twelve sequences are identified and delimited by sedimentary discontinuities. The lithostratigraphic division adopted in this study is synchronous with the coastal aggradation charter of Haq \& al. (1987 \& 1988) and Rioult \& al. (1991). The correlations on a regional scale, between different Jurassic lithostratigraphic units is possible, whose the major factors controlling the evolution of intraplate basins are climate and eustatism.
\end{abstract}

Keywords: Sequential stratigraphy, discontinuity, eustatism, Jurassic, Middle Atlas, Morocco

\section{Résumé}

L'évolution jurassique du Moyen Atlas central est régie par divers facteurs (climatiques, eustatisme). L'utilisation des concepts de la stratigraphie séquentielle dans les régions de Tagnamas et de Boulemane nous a permis d'interpréter la géométrie et l'enchaînement vertical des 
ensembles sédimentaires. Douze séquences sont distinguées et délimitées par des discontinuités sédimentaires. Le découpage lithostratigraphique adopté dans cette étude est synchrone avec la charte d'aggradation côtière de Haq et al. (1987 et 1988) and Rioult et al. (1991). Les corrélations à une échelle régionale, entre les différentes unités lithostratigraphiques du Jurassique est possible, dont les facteurs majeurs de contrôle de l'évolution de ces bassins intraplaques sont le climat et l'eustatisme.

Mots-clés : Stratigraphie séquentielle, discontinuité, eustatisme, Jurassique, Moyen Atlas, Maroc

\section{Introduction}

Le Jurassique du Moyen Atlas central est représenté par des dépôts très variés (épaisseurs et faciès) auxquels sont subordonnés divers types de discontinuités d'échelles différentes, organisés en corps sédimentaires. Pour retracer l'évolution de la région étudiée, durant le Lias et le Dogger, nous avons choisi deux exemples qui sont situés dans les régions de Tagnamas (coupe Ait Watfil) et de Boulemane (coupe Récifa), dont les âges sont complémentaires à compter du Domérien jusqu'au Bathonien. Dans la première coupe le Lias, l'Aalénien et le Bajocien inférieur sont bien représentés, les lithologies sont variées et les discontinuités sont bien exprimées. Dans la deuxième coupe le Bajocien et le Bathonien sont très developpés, les dépôts sont diversifiés et les hiatus de sédimentation sont de règle.

\section{Cadre géographique et géologique}

Le Moyen Atlas, qui fait partie du domaine des chaînes atlasiques oriental (Michard, 1976), est limité au nord par la plaine de Saïs et le front de la nappe rifaine, au Nord-Est par le bassin de Guercif, à l'Est et au Sud-Est par la dépression de la Moulouya et à l'Ouest par le Maroc central ( Fig.1).

Le Moyen Atlas est organisé en deux ensembles structuraux séparés par l'accident nord-moyen-atlasique (Choubert, 1967):

- le Causse moyen atlasique, composé essentiellement de carbonates néritiques du Lias inférieur et moyen, est structuré en plateaux étagés qui reflètent une organisation en blocs basculés ;

- le Moyen Atlas plissé est organisé en rides anticlinales (ride de Tichoukt, ride des Marmoucha,...) à ossature liasique que séparent de larges dépressions synclinales (Skoura, El Mers, Marmoucha...) à remplissage toarço-aaléno-bajocien. 


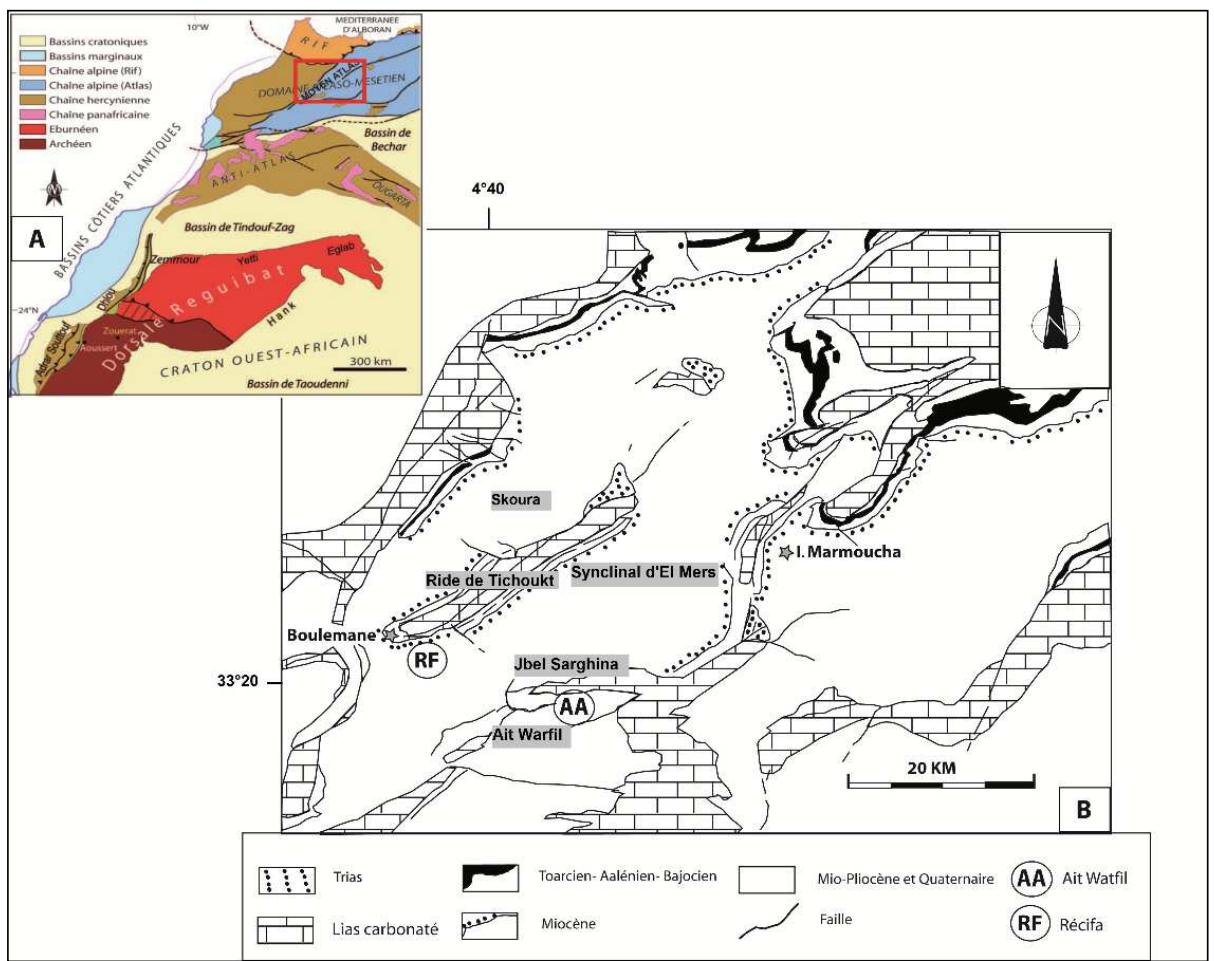

Figure 11: Localisation de la zone d'étude. A- Position du Moyen Atlas par rapport aux différents domaines structuraux du Maroc. B- Carte géologique simplifiée de la région étudiée.

\section{Méthodologie}

L'originalité de ce travail réside dans sa démarche multidisciplinaire faisant appel à des méthodes, et approches complémentaires. Il s'agit de :

- $\quad$ une approche classique de terrain, basée sur l'observation, le relevé de coupes et la récolte systématique de faune ;

- une approche lithostratigraphique permettant une définition précise des formations dont certains sont mal connu et s'étagent depuis le Domérien jusqu'au Bathonien supérieur ;

- $\quad$ une approche sédimentologique, basée sur la description des bancs jusqu'au niveau du microfaciès et des figures sédimentaires ainsi que le repérage des discontinuités ;

- une approche biostratigraphique permettant l'établissement d'un cadre chronostratigraphique fiable. Cette approche a permis de reconnaître toutes les zones et les sous zones du Toarcien, Aalénien et Bajocien du secteur d'étude;

- une approche basée sur l'analyse et la stratigraphie séquentielle : méthode synthétique qui intègre plusieurs disciplines (Sédimentologie, 
tectonique, paléontologique,....), afin d'établir un cadre chronostratigraphique à l'échelle globale.

Pour suivre l'évolution jurassique de cette partie du Moyen Atlas central, deux coupes sont levées: la coupe Ait Watfil le long de Jbel Sarghina et la coupe Récifa au terminaison périanticlinal de la Ride de Tichoukt.

\section{La région d'Aït Watfil (la coupe AA)}

La coupe AA est située dans la région de Tagnamas au Nord immédiat d'Aït Watfil, encadrée par le synclinal Ahlas où affleure largement le calcaire corniche et Jbel Serghina monoclinal à ossature liasique. Les dépôts toarciens sont représenté par des marnes, barre calcaire et marnocalcaires, quant au Bajocien, il est représenté par les marno-calcaires à Cancellophycus et les marnes de Boulemane (Fig. 2).

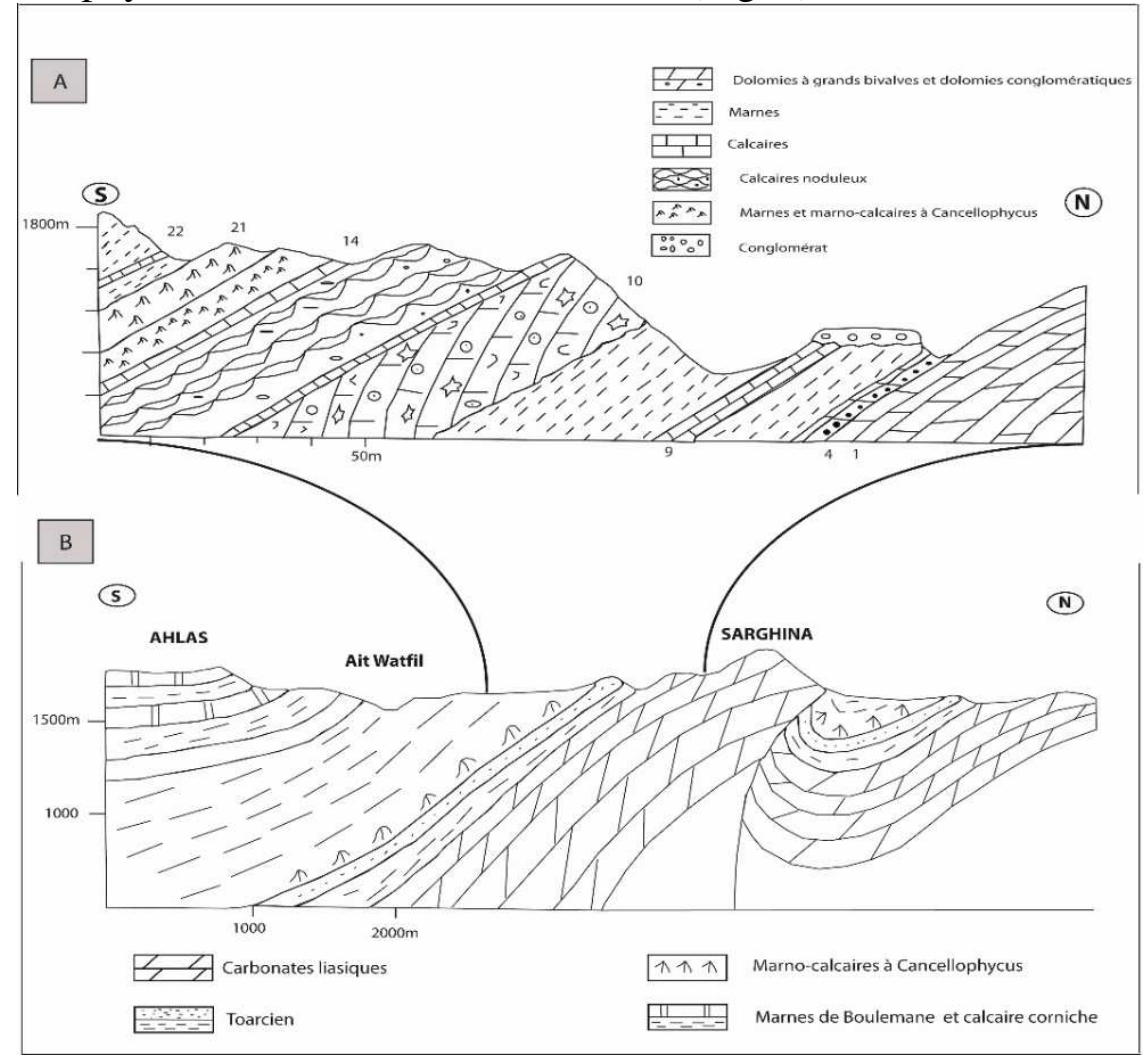

Figure 12 : Structure de la région d'Ait Watfil.

A- Coupe à travers la ride de Sarghina. B- Lias-Dogger d'Ait Watfil.

\section{Les grands ensembles lithostratigraphiques}

Les dépôts liasiques et bajociens de la coupe AA sont très variés sur le plan faciologique ils peuvent être subdivisés en cinq ensembles lithostratigraphiques (Fig.3) : le premier carbonaté, le deuxième 
essentiellement marneux, le troisième calcaire, le quatrième marno-calcaires et le cinquième marneux.

\section{Le premier ensemble lithostratigraphique}

Il est carbonaté avec des bancs décimétriques à grands bivalves (Mégalodon, Lithiotis-Cochlearites, Pecten et Pholadomya) et daté du Lias moyen (Domérien).

\section{Le deuxième ensemble lithostratigraphique}

Essentiellement marneux subdivisé en trois termes : marneux à foraminifères (Miliolidé, Siphovalvulina sp. et Haurania deserta), marnocalcaires et calcaires noduleux à bélemnites, gastéropodes, bois fossiles, ammonites (Dactylioceratidae) de la zone Polymorphum d'âge Toarcien inférieur (Elmi, 1994).

\section{Le troisième ensemble lithostratigraphique}

Il est totalement calcaire correspond à une barre décamétrique, organisée en bancs pluridécimétriques de calcaire grenu bioclastique, beige à stratifications obliques (de texture Grainstone voire Packstone au sommet) à Hildocéras sp., Coeloceras sp., Phymatoceras sp., Dactyloceratidae, Hammatoceras sp., Lytoceras sp., Planammatoceras sp., Harpoceras sp., Dumortieria sp., Catulloceras sp. et Pseudogrammoceras sp. de la zone à Bifrons d'âge Toarcien moyen (Elmi et Westermann, 1994).

\section{Le quatrième ensemble lithostratigraphique}

Il est représenté par une alternance marno-calcaire : les marnes grisverdâtres fossilifères à foraminifères (Lenticulina sp. Et Astracolus sp.), quant aux calcaires organisés en bancs décimétriques caractérisés par les Céphalopodes (bélemnites et ammonites: Phylloceras sp., Strigocéras sp., Paraeoppelia sp., Bradfordia sp., Calliphyllocéras sp., Emileia sp. et Stephanoceratidae) représentent la zone à Laeviuscula et (Sonninia sp., Skirrocéras sp., Kumatostephanus sp., Emileia sp., Dorsetensia sp.) représentent la zone à Sauzei. Ce terme marque le passage de la zone à Laeviuscula à la zone Sauzei du Bajocien inférieur (Sadki et Sandoval, 1993).

Notons que le Toarcien supérieur et la base d'Aalénien sont pelliculaires et mal daté.

\section{Le cinquième ensemble}

Il est marneux à foraminifères (Lenticulina munsteri, Dentalina bicornis et D. pseudocommunis) (Boutakiout, 1993) avec deux bancs pluridécimétriques de calcaires argileux à gastéropodes, bivalves et 
ammonites (Stéphanoceratidae) qui marque le passage de la zone Sauzei à la zone Humphriesianum du Bajocien inférieur.

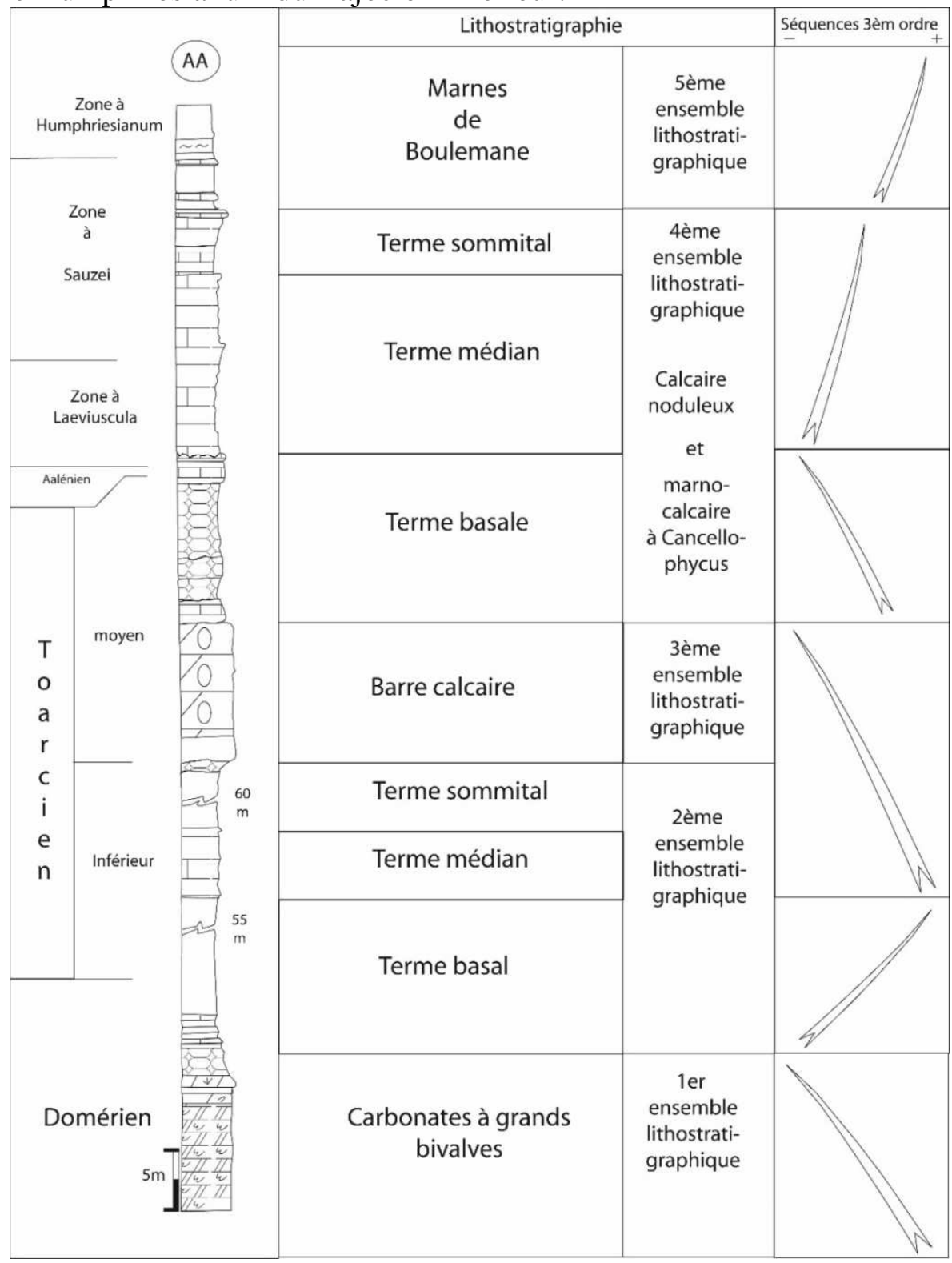

Figure 13 : Evolution sédimentologique du Lias-Dogger de la coupe AA

\section{Les discontinuités sédimentaires}

Sur le versant sud du Jbel Sarghina, les marno-calcaires à Cancellophycus bajociens reposent sur l'Aalénien supérieur très condensé, ce dernier recouvre un Toarcien élevé mal daté, ces lacunes de sédimentation qui marquent le passage Lias-Dogger se matérialisent par des discontinuités 
sédimentaires d'importance régionale. Une autre discontinuité sédimentaire majeure, datée du Domérien, sépare des faciès de plate-forme interne de ceux de milieux ouverts.

En plus de ces discontinuités d'échelle régionale, les discontinuités mineures sont fréquentes dans les dépôts jurassiques de la région d'Aït Watfil, parmi celles-ci nous avons reconnus et définis les types suivants : le diasthème, les changements brusques de la lithologie, le joint marneux, la surface irrégulière, bioturbée, ferrugineuse, encroûtée à galettes algaires et la surface de ravinement.

L'étude de la coupe AA, qui appartient à la marge méridionale du bassin jurassique du Moyen Atlas central est sous contrôles eustatique et tectonique qui ont contribué à l'élaboration de corps sédimentaires dont la composition ainsi que la polarité soulignent le caractère instable de cette bordure.

\section{La région de Récifa (la coupe $\mathbf{R F}$ )}

La région de Récifa est située au Sud immédiat de Boulemane, forme le versant Sud-est de la terminaison péri-anticlinale de la ride de Tichoukt. Dans cette région affleure bien les dépôts du Dogger qui sont représentés par les marnes de Boulemane et des carbonates qui ont subi des épisodes de dolomitisation précoce et/ou tardive, de dédolomitisation et de cimentation multiples.

La succession des gorges de Récifa est caractérisée par trois barres carbonatées décamétriques à pluridécamétriques formant des corniches bien repérables dans le paysage séparées par des dépôts marneux et marnocalcaires (Fig.4). Elles sont connues sous différents qualificatifs : calcaire corniche de Colo (1961-64) ; calcaire corniche inférieur et calcaire corniche supérieur de Du Dresnay (1963); complexe I et complexe II de Choubert et Faure-Muret (1967) (pour les deux premières barres) et barre dite des "troglodytes" (pour la troisième) Colo (1961-64). 


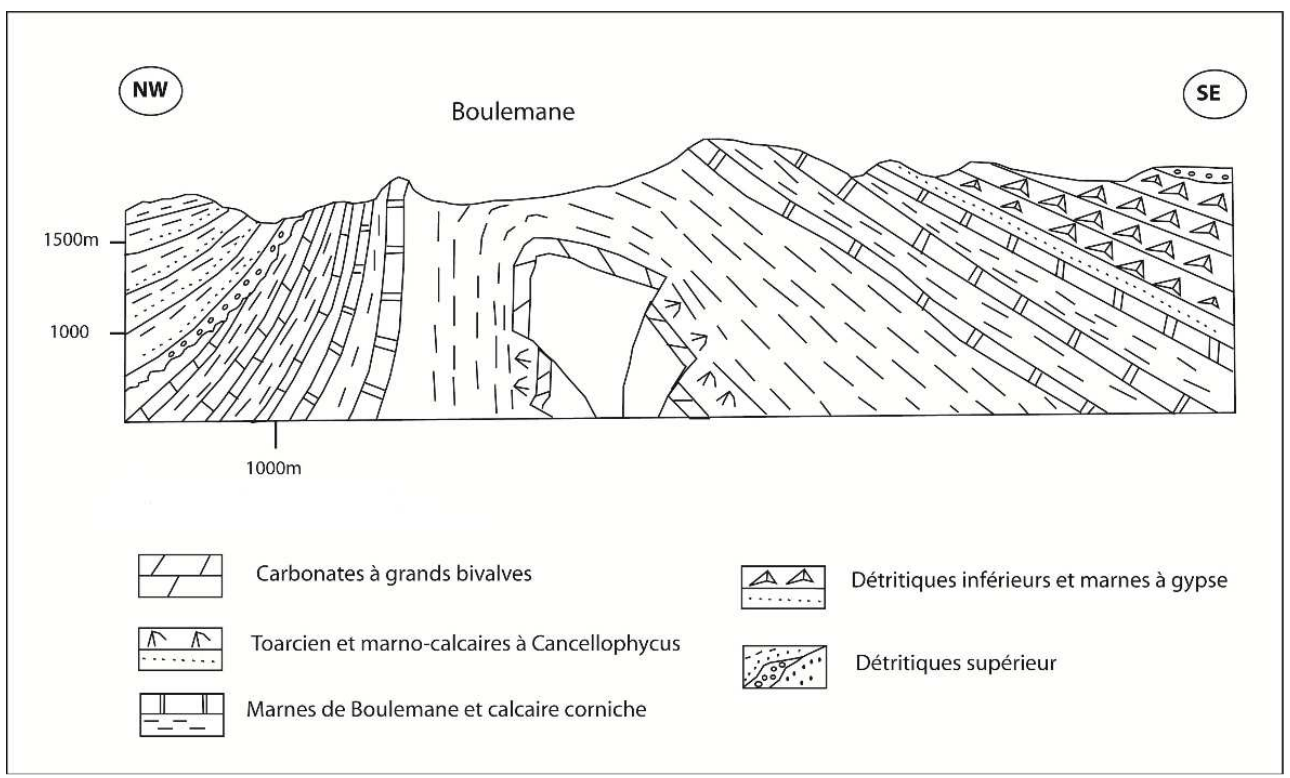

Figure 14: Structure de la région de Boulemane.

\section{Les grands ensembles lithostratigraphiques (Fig.5)}

\section{Le premier ensemble lithostratigraphique}

Correspond au sommet des marnes de Boulemane représenté par des marnes grises à la base et chargés par quelques bancs calcaires au sommet.

Les marnes ont fourni des Ostracodes et des foraminifères (Textularia agglutinans, T. Jurassica, Lenticulina pseudocrepidula, Eoguttulina polygona, Dentalina gumbelei) (Boutakiout, 1993); où s'intercalent des bancs décimétriques de calcaire argileux bioclastiques à bivalves (Plagiostoma sp., Ctenostreon sp., Pecten sp. et Pholadomya sp). Ils sont succèdés par deux croûtes centimétriques de calcaire bioclastique (de texture) packstone à brachiopodes, bryozoaires, gastéropodes, polypiers et échinodermes.

\section{Le deuxième ensemble lithostratigraphique}

Correspond à la première barre carbonatée, totalement dolomitisée. Il est organisé en bancs plurimétriques interrompu par des joints décimétriques d'argilites jaunâtres. Ces bancs sont coiffés par du calcaire massif (de texture packstone) à gastéropodes, bivalves (Pecten sp., Plagiostoma sp.) et foraminifères dont le sommet est très bioturbé riche en polypiers et couronné par une surface encroutée.

\section{Le troisième ensemble lithostratigraphique}

C'est une alternance plus ou moins régulière, dont la base est riche en calcaires. La phase carbonatée est représentée par des calcaires de textures 
variées et de dolomies qui résultent de dolomitisations précoce et tardive. Cet ensemble peut être subdivisé en trois termes : le terme basal est marqué par un grand développement des coraux, deux niveaux de calcaires oolithiques et un niveau de dolomies précoces; le terme médian est caractérisé par les calcaires oolithiques qui sont affectés par une dolomitisation précoce ; quant au terme sommital il est plus marneux.

\section{Le quatrième ensemble lithostratigraphique}

Correspond à la deuxième barre carbonatée, totalement dolomitisée et organisée en bancs pluridécimétriques à plurimétriques, elle débute par un niveau bioclastique et noduleux comme elle montre au sommet des passées oolithiques à stratifications obliques.

\section{Le cinquième ensemble lithostratigraphique}

Représenté par des marnes grises chargées en calcaires essentiellement bioclastiques. Il est marqué, à la base par deux bancs plurimétriques de calcaires à oolithes partiellement ou totalement dolomitisés, dans sa partie médiane se développent des passées de marnes versicolores, le sommet est caractérisé par des passées de calcaires bioclastiques à oolithes ainsi que par un banc plurimétrique de calcaire totalement dolomitisé.

\section{Le sixième ensemble lithostratigraphique}

Correspond à la troisième barre. Elle est composée d'un calcaire oolithique riche en quartz détritique totalement dolomitisé. Cette barre dite des "troglodytes" (Colo, 1961-64), est organisée en bancs pluridécimétriques à plurimétriques.

\section{Le septième ensemble lithostratigraphique}

Débuté par des marnes grises qui sont surmontées par la succession suivante : une dolomie oolithique grise, une dolomie laminitique, une brèche de tempête et une dolomie à oolithes et bivalves. Cette dolomie correspond à une dolomicrosparite de texture idiotopique imbriquée bioturbée à quartz détritique et lithoclastes. Le terme ultime de cet ensemble est représenté par des marnes versicolores à charbons, à ossements dinosauriens et à évaporites. 


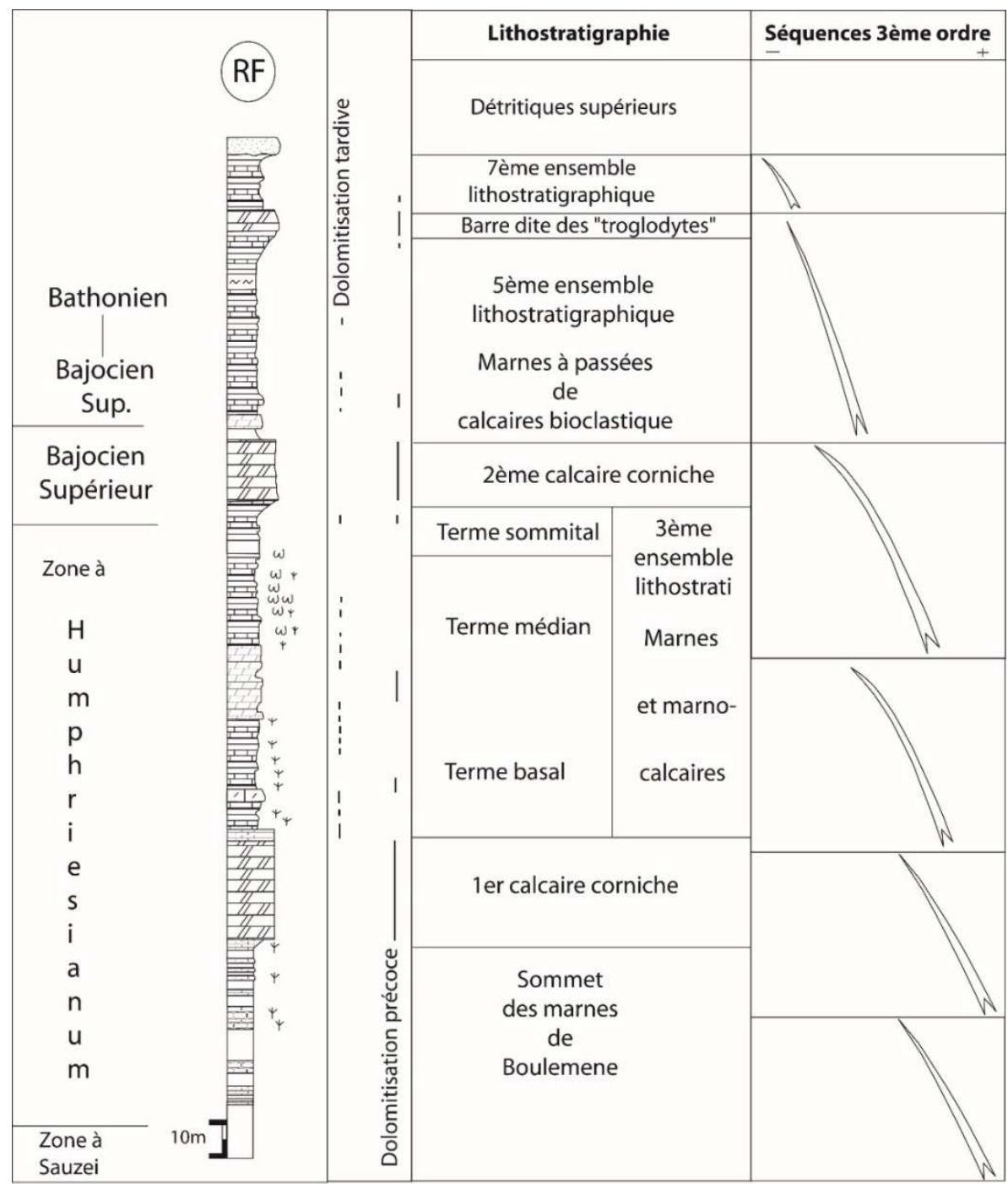

Figure 15: Evolution sédimentologique du Dogger de la coupe RF.

\section{Éléments de datation}

La succession des gorges de Récifa est représentée par des dépôts bioclastiques très variés, partiellement dolomitisés. Ils renferment des fossiles essentiellement benthiques, qui ne montrent pas de marqueurs biostratigraphique (Benjelloun, 1994). Pour pallier ce manque de données, nous nous sommes basés sur les données biostratigraphiques, relatives aux régions voisines :

- Dans la région d'Achlouj, de position méridionale, la première barre carbonatée est datée du Bajocien moyen (zone à Hmphriesianum).

- L'équivalent de la deuxième barre carbonatée de la succéssion des gorges de Récifa est daté au col de Souiguer, située plus au SE, du Bajocien 
supérieur (zone à Subfurcatum) par la présence de Telermoceras coronotö̈des (Du Dresnay, 1963).

- dans le synclinal d'El Mers, localisé au SE de Boulemane, la "formation d'El Mers" ou "couches d'El Mers", élément des détritiques inférieurs (Fedan, 1989), est daté du Bathonien moyen par la découverte de Cadomites cf. bremeri TSERETELI (Fedan, 1989). ces détritiques sont antérieurs aux marnes à gypse qui surmontent la succession des gorges de Récifa.

En tenant compte de toutes ces données, d'échelles locale et régionale, nous avons proposé le découpage chronostratigraphique suivant :

$\checkmark \quad$ Les $1^{\text {er }}, 2^{\text {ème }}$ et $3^{\text {ème }}$ ensembles lithostratigraphiques sont attribués au Bajocien (zone à Humphriesianum).

$\checkmark \quad$ le sommet du $3^{\text {ème }}$ ensemble ainsi que le $4^{\text {ème }}$ ensemble sont rapportés au Bajocien supérieur.

$\checkmark \quad$ les $5^{\text {ème }}, 6^{\text {ème }}$ et $7^{\text {ème }}$ ensembles sont datés du Bajocien supérieur voire Bathonien basal.

\section{Les discontinuités sédimentaires}

Les marnes de Boulemane et la succession des gorges de Récifa montrent des caractéristiques faciologiques variées, les dépôts sont agencées aux différentes échelles, en séquences négatives et ont enregistré divers types de discontinuités sédimentaires. Parmi celles-ci nous avons définis: une discontinuité sédimentaire majeure, sépare des faciès de plate-forme interne (la première barre) de ceux de milieux ouverts (alternance marno-calcaires) daté de Bajocien moyen au Bajocien supérieur, une autre discontinuité intraBajocien supérieur limitant la deuxième barre et l'alternance marnocalcaires.

L'étude la coupe de Récifa permet de faire une mise au point sur les formations du Bajocien (les marnes de Boulemane et les calcaires corniches) dans leur localité-type qu'est Boulemane, en plus elle retrace la dynamique bajocienne du bassin jurassique du Moyen Atlas, en particulier au droit d'une ride mobile (Tichoukt). Ces bouleversements paléogéographiques sont guidés par les trois paramètres principaux: l'eustatisme, la tectonique et le taux de sédimentation ainsi que le rigime climatique qui régit le developpement de la dolomitisation.

\section{La stratigraphie séquentielle}

Pour retracer l'évolution de la région étudiée durant le Lias et le Dogger, nous avons utilisé les concepts de la stratigraphie séquentielle qu'on l'applique suivant les variations brutales matérialisées par des surfaces de discontinuités sédimentaires ou un niveau durci Hard ground. 


\section{Séquences de dépôts}

L'analyse des dépôts des deux coupes AA et RF, dont l'âge s'échelonne du Domérien au Bathonien, a permis de reconnaître et définir douze séquences de dépôt, délimitées par des discontinuités sédimentaires d'extension régionale et sont numérotées de SD1 à SD12 :

- $\quad$ La séquence de dépôt SD1 (Domérien), elle est représentée par les carbonates à grands bivalves qui sont des dépôts tidaux.

- La séquence de dépôt SD2 (Domérien terminal- Toarcien inférieur), elle débute par des carbonates noduleux et se termine par des marnocalcaires à valeur de cortège de haut niveau, d'où la stabilité du niveau marin.

- La séquence de dépôt SD3 (Toarcien inférieur et moyen), elle comprend à la base des marnes à Céphalopodes qui peuvent être assimilées à un cortège transgressif. Au sommet, il s'agit d'une barre carbonatée, d'allure progradante, composée de dépôts dynamiques qui sont représentés essentiellement par des calcaires encrinitiques et oolithiques.

- Les séquences de dépôt SD4, SD5 et SD6 (Toarcien moyen-? Toarcien supérieur et ? Aalénien), ces trois séquences montrent les mêmes compositions et organisation interne. Elles débutent par des marnes à concrétions calcaires ou par des marno-calcaires que l'on peut interpréter comme un cortège transgressif, elles se terminent par des calcaires noduleux ou par des marno-calcaires très bioclastiques à valeur de cortège de haut niveau. Notons que ces trois séquences sont marquées par la fréquence des Céphalopodes ainsi que par le caractère noduleux des dépôts qui attesterait d'une instabilité du tréfond.

- La séquence de dépôt SD7 (Aalénien-zone à Laeviuscula), elle montre une semelle aalénienne qui est composée de calcaires noduleux. Les dépôts sont représentés par les marno-calcaires à Cancellophycus : les bancs calcaires stratocroissants à la base et stratodécroissants au sommet d'où la succession des cortèges transgressif et de haut niveau.

- La séquence de dépôt SD8 (zone à Sauzei). Elle présente une organisation similaire à la séquence précédente. Il s'agit de marnes et marnocalcaires à Cancellophycus : les bancs calcaires sont stratodécroissants à la base et stratocroissants au sommet. Cette organisation verticale des dépôts reflète la succession des cortèges transgressifs et de haut niveau. Cette séquence et celle qui la précède traduisent un approfondissement notable des milieux de dépôts, bien que la bathymétrie a connu des fluctuations. Cette remontée eustatique va s'affirmer durant les zones à Sauzei et à Humphriesianum lors du dépôt des marnes de Boulemane (séquence SD9). 


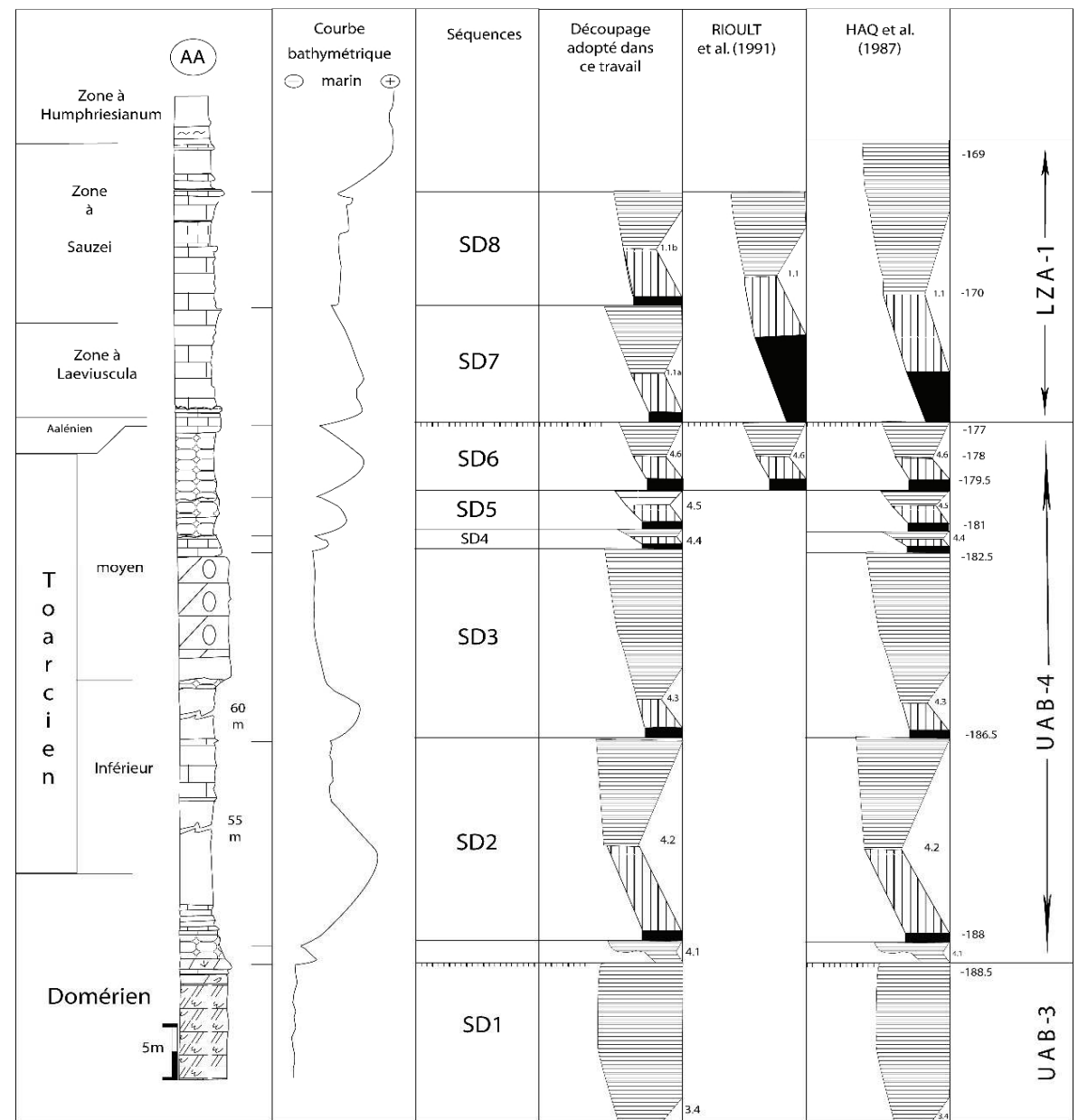

Figure 16: Courbe bathymétrique, successions sédimentaires et comparaison avec les courbes d'aggradation côtière

- $\quad$ la séquence de dépôt SD9 (zones à Sauzei et à Humphriesianum), cette séquence est la plus dilatée, englobe les marnes de Boulemane et le premier calcaire corniche. Le sommet des marnes de Boulemane est chargé en niveaux carbonatés bioclastiques à faune benthique et en passées de marnes versicolores ce qui traduit des fluctuations du niveau marin ainsi qu'une amorce de la diminution de la bathymétrie. Cette séquence est clôturée par un niveau bréchifié.

- La séquence de dépôt SD10 (zone à Humphriesianum), elle est composée essentiellement de marno-calcaires à polypiers où s'intercalent deux passées oolithiques et plusieurs niveaux noduleux. Cette séquence est coiffée par une surface de ravinement importante.

- $\quad$ La séquence de dépôt SD11 (zone à Humphriesianum et Bajocien supérieur), elle débute par deux bancs épais, de texture jointive, oolithiques à la base ; ils traduisent une chute eustatique qui sont assimilés à un prisme de 
bordure de plate-forme. Viennent ensuite des marno- calcaires à polypiers et à Céphalopodes (bélemnites) dont les bancs calcaires montrent une certaine stratodécroissance; on assiste là à une élévation du niveau marin et par conséquent à l'instauration d'un cortège transgressif.

- La séquence de dépôt SD12 (Bajocien supérieur-Bathonien inférieur), à la base les marnes sont armées de deux bancs épais : le premier, totalement dolomitisé (dolomitisation précoce), montre des traces d'évaporites ; le deuxième, partiellement dolomitisé, est composé de dépôts dynamiques. La base de cette séquence peut être assimilée à un prisme de bordure de plate-forme. Viennent ensuite des marnes à rares intercalations bioclastiques, qui correspondent à un cortège transgressif. Après quoi, le niveau marin se stabilise d'où l'apparition de marnes qui sont chargées en calcaires et qui montrent des passées de marnes versicolores, ce qui annonce le développement du cortège de haut niveau. Ce dernier se poursuit par la troisième barre carbonatée dite "'barre de troglodyte"; elle est composée de calcaires oolithiques qui sont affectés par une dolomitisation précoce.

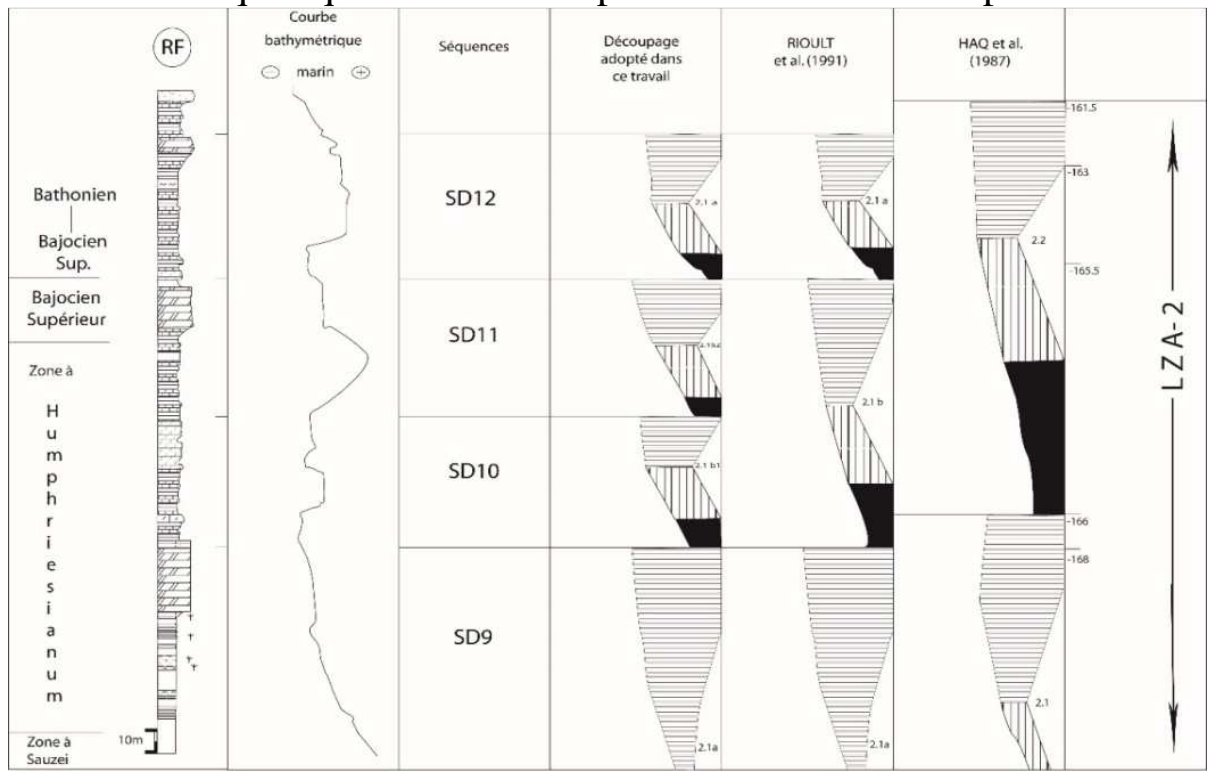

Figure 17: Courbe bathymétrique, successions sédimentaires et comparaison avec les courbes d'aggradation côtière

Comparaison avec la courbe d'aggradation côtière de HAQ et al. (1987 et 1988) et RIOULT et al. (1991)

La courbe d'aggradation côtière de Haq et al. (1987 et 1988) diffère peu, pour le Dogger, de la courbe établie par Rioult et al. (1991) pour le Jurassique de Normandie. La comparaison de la courbe bathymétrique locale, que nous avons établie pour le Jurassique du Moyen Atlas central 
avec ces chartes nous a permis de faire les observations suivantes (Fig.6 et Fig.7):

$>\quad$ la séquence SDI coïncide avec le cycle eustatique UAB-3.4

$>\quad$ les séquences SD2, SD3, SD4, SD5 et SD6 correspondent respectivement aux cycles UAB-4.2, UAB-4.3, UAB-4.4, UAB-4.5 et UAB4.6. Notons que la séquence SD1 bis, que nous n'avons pas définie, a pour équivalent le cycle UAB-4.1.

$>\quad$ l'ensemble formé par les séquences SD7 et SD8 peut être mis en parallèle avec le cycle LZA-1.1; ce qui implique l'existence d'un cycle eustatique supplémentaire dans le Bajocien inférieur (zones à Laeviuscula et à Sauzei).

$>\quad$ la séquence SD9 coïncide avec le cycle LZA-2.1a.

$>\quad$ l'ensemble formé par les séquences SD10 et SD11 peut être mis en parallèle avec le cycle LZA-2.1b; ce qui implique l'existence d'un cycle eustatique supplémentaire dans le Bajocien supérieur.

$>\quad$ la séquence SD12 correspond au cycle LZ-2.2a.

\section{Interprétation}

Selon notre conception, on note un remarquable synchronisme entre le découpage que nous avons proposé et celui établie par Haq et al. $(1987,1988)$ et modifié légèrement par Rioult et al. (1991) pour le Dogger. Cependant, il faut souligner la mise en évidence de séquences de dépôt, et par conséquent de cycles eustatiques supplémentaires notamment au Bajocien inférieur (zones à laeviuscula et à Sauzei : SD7 et SD8 équivalentes de LZA-1.1) et au Bajocien supérieur (SDI0 et SD11 équivalentes de LZA2.1b). De plus, il faut remarquer que les deux discontinuités majeures de type 1 des chartes, datées de $-188,5 \mathrm{Ma}$ et de $-177 \mathrm{Ma}$, sont bien exprimées dans le Moyen Atlas central. Elles se situent respectivement aux passages du Lias moyen au Lias supérieur et du Toarcien au Dogger.

\section{Conclusion}

Le découpage lithostratigraphique au Jurassique du Moyen Atlas central est synchrone avec les courbes d'aggradation côtière de Haq et al. (1987, 1988) et Rioult et al. (1991) suivant les régions d'Ait Watfil et Récifa. Donc en plus des variations eustatiques, l'architecture des corps sédimentaires est régie également par la mobilité du substratum, le taux de sédimentation, la tectonique et le climat qui n'ont que des influences locales. La signature composite de tous ces facteurs se marque dans les sédiments sous forme : des changements de faciès, des variations de puissance, de la diversité des lithologies, de la fréquence des marqueurs de la déformation synsédimentaire et de la présence de nombreux hiatus de la sédimentation. La stratigraphie séquentielle peut constituer un outil très performant de 
corrélation des séries stratigraphiques, d'interprétation de leurs agencements et de prévision de localisation des cortèges sédimentaires.

Ainsi, le Moyen Atlas central constitue un bon modèle pour l'étude des phénomènes généraux liés aux variations eustatiques et des phénomènes locaux dûs à la tectonique en blocs basculés.

\section{References:}

1. ALMERAS Y. et ELMI S. (1982) - Fluctuations des peuplements d'ammonites et de brachiopodes en liaison avec les variations bathymétriques pendant le Jurassique inférieur et moyen en Méditerranée Occidentale. Boll. Soc. Paleont. Ital., Modena, vol. 21, n²-3, p. 169188.4 fig., 3 pl.

2. BENJELLOUN F. (1994): Evolution jurassique du Moyen Atlas central : contrôles tectono-eustatique et climatique de la dynamique sédimentaire. Thèse $3^{\mathrm{èm}}$ cycle, Fac. Sci. Rabat, 378p.

3. BENSHILI K. (1989) - Lias-Dogger du Moyen Atlas plissé (Maroc), Sédimentologie, Biostratigraphie et Evolution paléogéographique. Docum. Labo. Géol. Lyon, n¹06, 1989, 285p.

4. BOUTAKIOUT M. (1990) - Les foraminifères du Jurassique des Rides sud-rifaines et des régions voisines (Maroc). Docum. Labo. Géol. Lyon. $\mathrm{n}^{\circ}$ 112, 1990, 247p.

5. CHOUBERT G. \& FAURE-MURET A. (1967) - Le Jurassique de la région d'El Mers-Skoura (Moyen Altas). In : Etudes sur le Jurassique du Moyen Atlas (Maroc). Notes el Mém. Serv. Géol. Maroc, $n^{\circ} 200$.

6. COLO G. (1961-64) - Contribution à l'étude du Jurassique du Moyen Atlas septentrional. 2 volumes. Note et Mém. Serv. Géol. Maroc, $\mathrm{n}^{\circ}$ $139 \cdot 226 \mathrm{p}$.

7. DELFAUD J. (1980) - Le contexte paléogéographique des séquences d'échelle moyenne dans le Jurassique aquitain. Bull. Soc. géol. France, (7), t. XXII, n ${ }^{\circ}$, p. 573-579.6 fig.

8. DU DRESNAY R. (1963) - Données stratigraphiques complémentaires sur le Jurassique moyen des synclinaux d'El Mers et de Skoura (Moyen Atlas Maroc). Bull. Soc. Géol., France, $7^{\circ}$ série, t.V, p. 883-900.

9. ELMI S. (1985) -corrélations biostratigraphiques et mégaséquentielles dans le Jurassique inférieur et moyen d'Oranie. Comparaisons avec les régions voisines. Ve Conf. Sci. Int. Marrakech, P.I.C.G. 183.

10. FEDAN B. \& LAADILA M. (1984) - évolution séquentielle du Jurassique du Moyen-Atlas. Le Lias du Jbel Amrar (SSE de Boulemane) : un exemple type de séquence d'accrétion 
(klupfelienne). Note présentée aux Journées géol. et min. marocaines (1984) dans Mines, Géologie et Energie, Rabat.

11. FEDAN B. (1989),-Evolution géodynamique d'un bassin intraplaque sur décrochements: le Moyen Atlas (Maroc) durant le MésoCénozoïque. Trav. Inst. Sci., Rabat série Géol. Géogr. Phys. n ${ }^{\circ} 18$, $142 p$.

12. GABILLY J., CARIOU E. et HANTZPERGUE P. (1985) - Les grandes discontinuités stratigraphiques au Jurassique : témoins d'évènements eustatiques, biologiques et sédimentaires. Bull. Soc. géol. France, Paris, (8), t. I, n³, p. 391-401, 6 fig., 1 tabl.

13. HAQ B.U., HARDENBOL J. and VAIL P.R. (1987) - The chronology of fluctuating sea-Ievel since the Triassic. Science, vol.235, pp.1156-1167.

14. HAQ B.U., HARDENBOL J. and VAIL P.R. (1988) - Mesozoïc and Cenozoïc chronostratigraphy and facies of sea-level change ln: Seatevel changes: an integrated approch, SEPM Special Publication $n^{\circ}$ 42, pp. 71-108.

15. RIOULT M., DUGUE O., JAN DU CHENE R., PONSOT C., FILY G., MORON J.M. ET VAIL R. (1991) - Outcrop sequence srratigraphy of the Anglo-Paris Basin, Middle to Upper Jurassic (Normandie, Maine, Dorset). Bull. centres Rech. Explor. Elf Aquitaine, 15, 1. pp. 101-194.

16. SADKI D. (1984) - Biostratigraphie et paléontologie des Ammonites de quelques gisements du Bajocien inférieur du Maroc, de France et du Portugal. Thèse $3 e$ Cycle, 191 p. 44 fig., 21 tabl. 6 pl.

17. SADKI D., FEDAN B. \& BENJELLOUN F. (1994) - Evolution géodynamique jurassique $\mathrm{du}$ domaine des chaînes atlasiques marocaines, illustrée par la transversale Boulemane-MideltErrachidia Aalénien and Bajocien working groups meeting; Marrakech 25-31 May 1994. Fieldtrip Guide- Book, pp. 48-95.

18. SANDOVAL J. (1986) -Middle Jurassic Haploceratidea (Ammonitina) from the subbetic zone (South Spain). Géobios, Lyon, $n^{\circ} 19$, fasc. 4, p. 435-463, 11 fig., 3 pl.

19. VAIL P.R. COLIN J.P. CHENE R.J. KUCBLEY J., MEDtAVILLA F. \& TRJFJLIEFF V. (1987) -La stratigraphie séquentielle et son application aux corrélations chronostratigraphiques dans le Jurassique du bassin de Paris. Bull. Soc. Géal. France.(8), t, III. n 7, pp. 13011321.

20. Westermann, G.E.G. (1964) - The ammonite fauna of the Kialagvik Formation at Wide Bay, Alaska Peninsula. Part I, lower Bajocian (Aalenian). Bulletins of American Paleontology, Ithaca New York, 17, $n^{\circ} 216$ p. 325-503. 37 fig. , 33pl. 